\title{
Does 3D back contour changes following spinal fusion in children with idiopathic scoliosis?
}

\author{
John Thometz ${ }^{*}$ Xuecheng Liu, Channing Tassone \\ From 11th International Conference on Conservative Management of Spinal Deformities - SOSORT 2014 \\ Annual Meeting \\ Wiesbaden, Germany. 8-10 May 2014
}

\section{Background}

Oftentimes, patients find trunk asymmetry, including thoracic rib hump, shoulder level difference, more troubling than a significant Cobb angle, as topographical deformity can be readily visualized. Evaluating improvement in these back contour parameters lends to additional criteria by which to assess post-surgical improvement. Quantec raster stereography is recognized as an accurate and reliable tool for monitoring threedimensional back contour in patients with idiopathic scoliosis before and after surgery.

\section{Aims}

To investigate the 3D back contour changes in adolescent idiopathic scoliotic deformity from surgical intervention using Quantec imaging.

\section{Design}

In this prospective controlled study, 35 patients undergoing anterior and/or posterior spinal fusion were evaluated pre and post-operatively by Quantec Spinal Imaging System and radiography.

\section{Methods}

Mean age of patients 14.5 years, with mean follow-up duration 1.7 years. A reliable Quantec protocol was established previously in the literature, consisting of twelve parameters. Pre and post-operative parameters were analyzed by paired t-test evaluating the effects of spinal fusion on scoliosis deformity.

\section{Results}

Significant improvement was seen in thoracic Cobb angle from 52.9 to 19.6 degrees $(\mathrm{p}<.0001)$ and

Department of Orthopaedic Surgery, Medical College of Wisconsin, Milwaukee, WI, USA 